\title{
5 Implantation: Flap and Subdural Lead Placement
}

Implanted electrodes used in reported studies have generally been Resume (Medtronic) or Lamitrode (ANS) stimulating strips, both quadripolar electrode arrays (each contact $5 \mathrm{~mm}$ in diameter; inter-contact distance center-to-center $1 \mathrm{~cm}$ ); eightcontact paddles have been used in one failed trial(NCT00122915). Two strips can be positioned in parallel for wider coverage. The patient either lies down supine (Nguyen et al 1999, 2011) or in lateral decubitus (Park Bench) (Pirotte et al 2009) allowing horizontal orientation of the operative field and easy access to the contralateral limbs under the table.

\subsection{Reference Technique}

The first phase of the operation consists of identifying the CS on axial sections (Fig.5.1A-B), then plotting a trajectory starting at the CS in the zone corresponding to representation of the upper limb. This zone can be identified by the classical $\Omega$ shape (see Chapter 1) of the CS, or with reference to the posterior part of the superior frontal sulcus responsible for this particular shape of the CS. The trajectory entry point is defined so that the trajectory is perpendicular to the brain surface (Fig.5.1C); as explained elsewhere in this volume, many others opt for a parallel positioning and results are similar with the two approaches. A view perpendicular to the trajectory ("probe eye view" mode) is then selected to visualize all of the central region in order to more precisely choose the target according to cortical somatotopy. For example, in order to target the area of representation of the face, the target must be placed more laterally, in the posterior part of the inferior frontal sulcus (Fig.5.1D). Similarly, the area of representation of the lower limb is targeted by placing the target between the superior frontal sulcus and the midline.

The second phase of the operation consists of performing a circular craniotomy, about $4 \mathrm{~cm}$ (in other authors'experience up to $7 \mathrm{~cm}$ ) in diameter (length of the quadripolar lead) centered around the target point chosen at the previous step. It is important to ensure at each step that the craniotomy is precisely centered around the target point (Fig.5.2). 

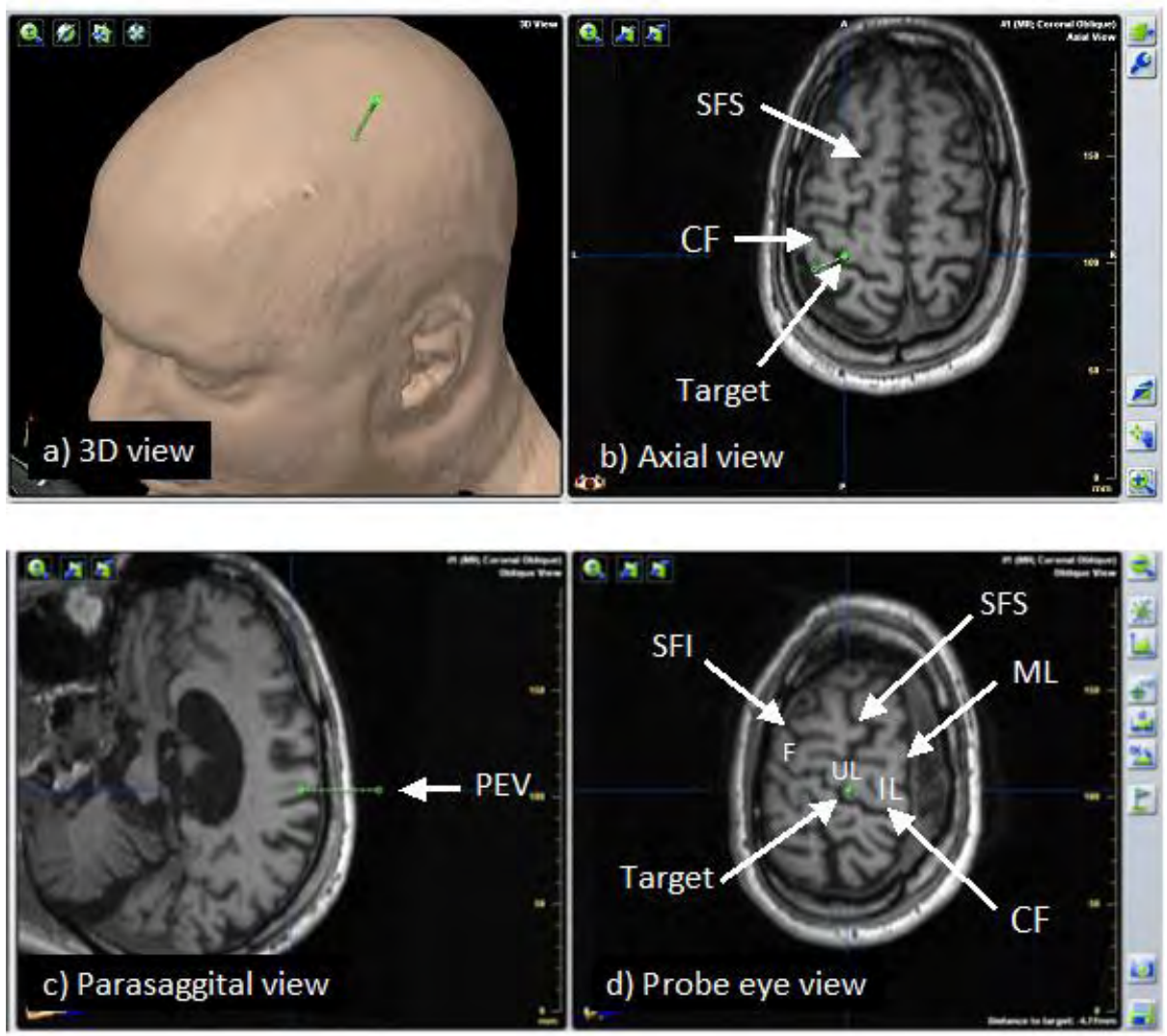

Fig. 5.1: Neuronavigation (Brainlab ${ }^{\circledR}$ ). The first step consists of localizing the central sulcus (CS) on an axial scan (b). The first target consists of the zone of representation of the upper limb situated posteriorly to the posterior part of the superior frontal sulcus (SFS). The Probe Eye View (PEV fig. $C$ and d) then allows easy identification of the various structures of the central region: CS: central sulcus, SFS: superior frontal sulcus, IFS: inferior frontal sulcus, ML: midline. The various functional zones of the motor cortex can be delineated in the axis of the trajectory (PEV). F: face, UL: upper limb, LL: lower limb. 


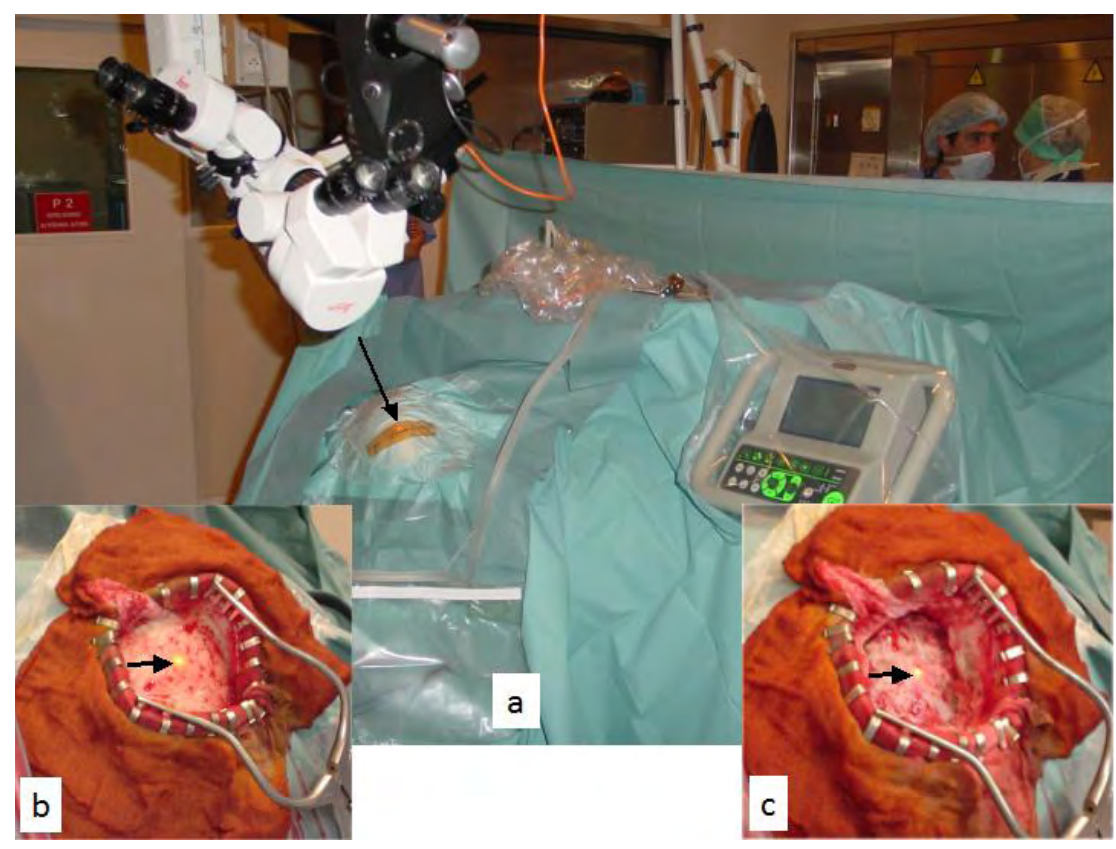

Fig. 5.2: Neuronavigation (Surgiscope ${ }^{\circledR}$ ). The previously determined target (Fig. 1) is represented by a laser beam (black arrow Fig. a). After performing the skin incision (Fig. b) and craniotomy (Fig. C), it is important to check that the craniotomy remains centered around the target (black arrow).

The third phase corresponds to intraoperative electrophysiological testing. Electrophysiological testing can be performed with a grid (comprising 16 electrodes in this case) or a quadripolar lead, which will then be used for chronic stimulation (Fig.5.3). The disadvantage of this second option is that the electrodes must be moved to different positions to ensure electrophysiological testing of all of the zone exposed by the craniotomy. We use classical self-retaining retractors to temporarily maintain the electrodes on the dural surface. M1 can be localized indirectly by studying the SSEPs obtained after stimulation of the median nerve in the wrist. Inversion of the N20/P20 potential indicates the position of the CS (Fig.5.4). Motor evoked potentials (Chapter 3) can be used to localize the zone of M1 corresponding to representation of the upper limb or face, as responses to cortical stimulation are recorded in the first interosseous space of the hand (Fig.5.4A) or in the labial commissure. Localization of the zone corresponding to the lower limb is more difficult, but, in a fairly large number of cases, this zone sometimes largely overlaps the superior part of the hemispheric convexity and can be investigated as described above. We initially used repeated stimulation to obtain MEPs (Fig.5.5C), which had the advantage of evoking muscle contractions that are clearly visible (Fig.5.5A-B) or recordable under classical general anesthesia (Fig.5.5C). However, this approach was associated with a risk of 
inducing seizures, as the amplitude of the response progressively increased during stimulation (Fig.5.5C). We currently prefer single-impulse stimulation (Fig.5.4E). In order to reduce the intensity of stimulation required to obtain MEPs, anesthesia is lightened by using BIS monitoring (Conte et al 2013) (Fig.5.5D).
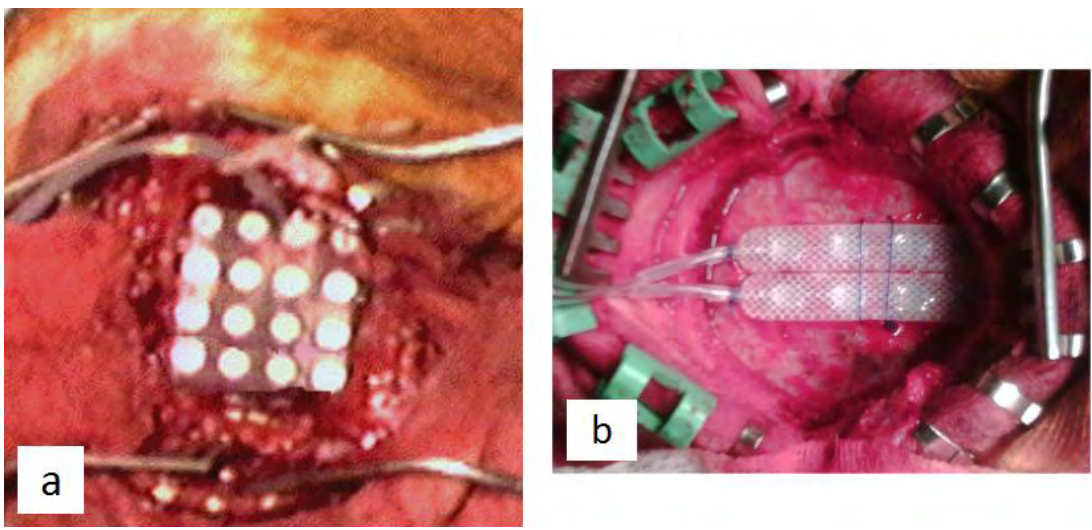

Fig. 5.3: Intraoperative electrophysiological testing. This study can be performed by using a grid (comprising 16 electrodes in this case) (Fig. a) placed on the dura mater or Resume leads, which will then be used for chronic stimulation (Fig. b). In this case, the electrode positions must be changed to test all of the exposed dural surface.

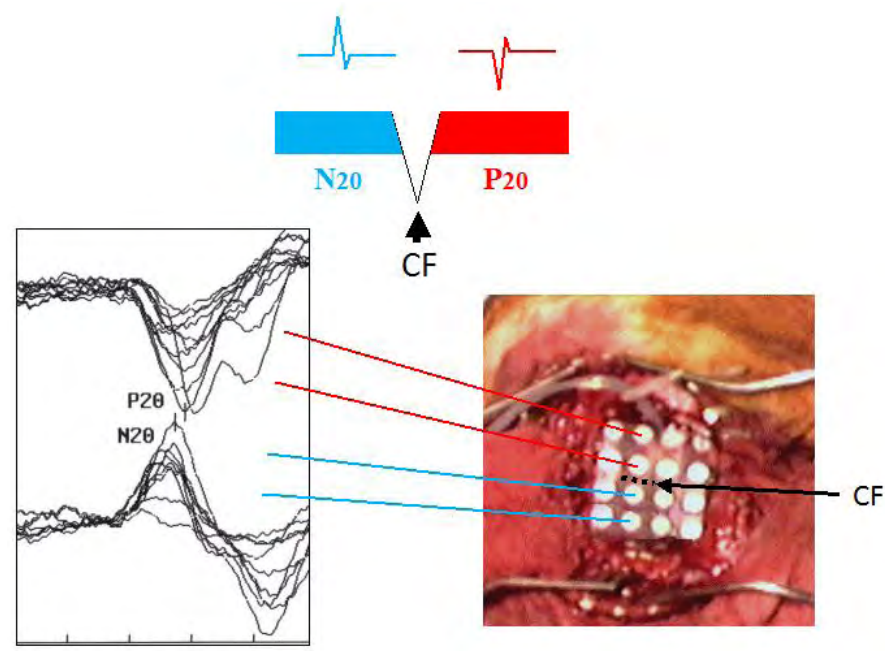

Fig. 5.4: Somatosensory evoked potentials. The signal evoked by stimulation of the median nerve in the wrist is recorded (after about 20 milliseconds) on all electrodes of the grid. The negative P20 potentials are recorded on electrodes placed anteriorly to the central sulcus (dotted line on the righthand Fig.) and the positive $\mathrm{N} 20$ potentials are recorded on electrodes situated posteriorly to the central sulcus, which confirms the position of the central sulcus. 

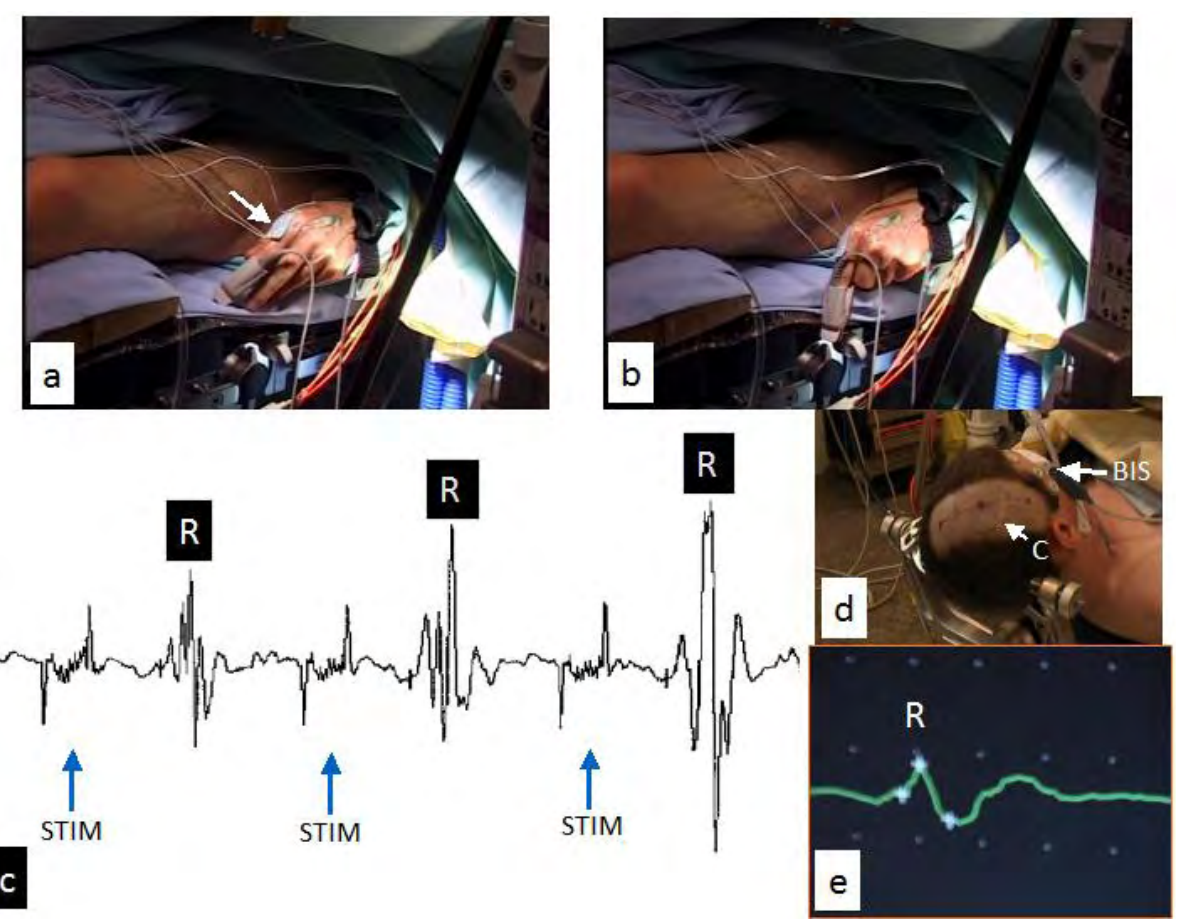

Fig. 5.5: Motor evoked potentials. Stimulation of electrodes placed anteriorly to the central sulcus induces motor responses, which confirm the position of the motor cortex. Motor responses can be observed clinically (Fig.s a and b) in response to repeated stimulation (Fig. C). In this case, stimulation at $16 \mathrm{Hertz}$ (STIM) induces motor responses whose amplitude increases progressively with stimulation (R). A high stimulation intensity facilitates clinically visible motor responses (Fig. b), but is associated with a risk of seizures. We currently prefer to use single-impulse stimulation, which is not associated with any risk of seizures, but which usually does not induce a clinically visible response. The response must then be recorded ( $R$ Fig. e) and the depth of general anaesthesia must be lightened. Management of the depth of general anaesthesia is facilitated by the use of BIS monitoring. The EEG signal recording electrode is placed on the patient's forehead (B Fig.s d). Figure $d$ also indicates the limits of the craniotomy (c).

No stimulation leads specifically designed for cortical stimulation are available at the present time. By analogy with spinal cord stimulation, which is also epidural, we use the same Resume leads (Medtronic, Minneapolis, USA) (Fig.5.3B). Other leads from other manufacturers are also adequate.

We decided to place stimulation leads perpendicularly to the CS, essentially to compensate for any placement errors that could be related to parallax error, as the sulci and fissures of the central region are easily recognized on relatively deep sections, but a parallax error may be observed on the surface (Nguyen et al 1999). On the other hand, by allowing stimulation on both sides of the CS, these leads can be used to stimulate the part of M1 situated in the anterior bank of the CS. The work by 
Holsheimer et al (2007) suggested that the best combination consisted of placing the cathode (negative pole) just anteriorly to the CS and the anode (positive pole) just posteriorly to the CS (Fig.5.6A).

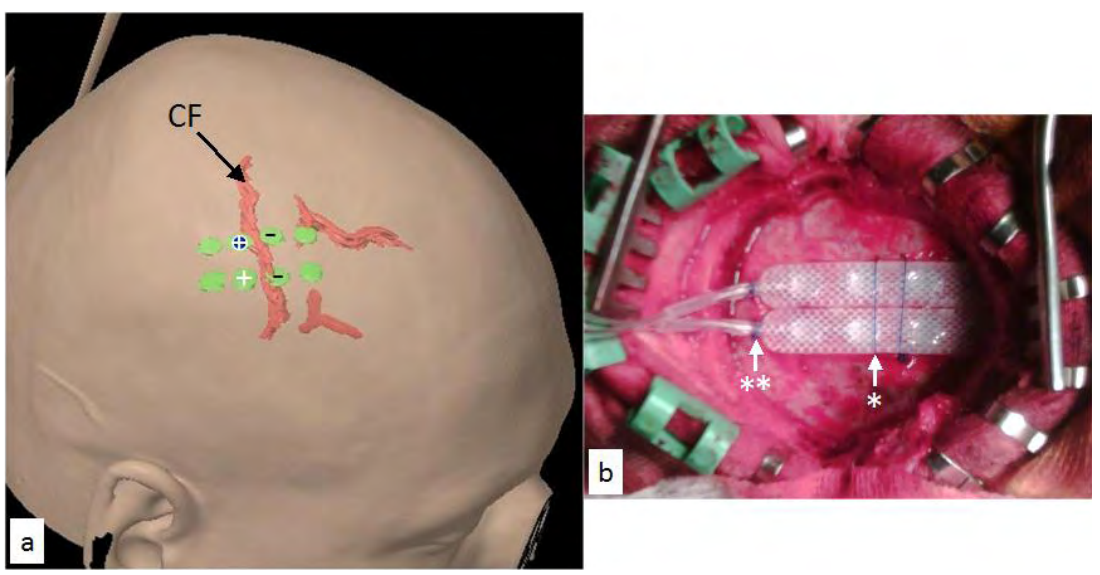

Fig. 5.6: Lead positions. We use two Resume 4-electrode leads placed in parallel and perpendicular to the axis of the central sulcus (CS). The optimal stimulation parameters consist of placing the cathodes (negative poles) anteriorly to the central sulcus (CS) and the anodes posteriorly to the central sulcus (Fig. a). Leads are sutured to the dura mater at 2 levels (Fig. b). A first suture comprises the two leads to limit lateral movements $\left({ }^{*}\right)$. The zone of connection of the lead with the extension is anchored by a second suture in order to limit anteroposterior movements $\left({ }^{\star *}\right)$.

The leads are then sutured to the dura mater (Fig.5.6B). We try to stabilize the anterior extremity of the leads by pushing them slightly underneath the edges of the craniotomy and the leads are anchored by a suture comprising the two leads, which limits the risk of lateral displacement. To avoid displacement along the long axis of the leads, the leads are sutured to the extension connector. Very careful hemostasis is highly recommended for avoiding epidural infectious or hemorrhagic complications. Full superficial denervation of the dura by bipolar coagulation of its surface may reduce the local (cranial) pain described by some patients when switching the stimulation on. The electrode must be fixed to the dura with very tight sutures to minimize the space between the electrode and the dura, in which scar tissue can develop and thereby increase the impedance of the stimulation and require higher voltage. One way to fix the electrode is by means of a single 3-0 silk suture every $5 \mathrm{~mm}$, with 2 sutures bridging the electrode in order to maintain it against the dura. Some authors drill a groove at the posterior margin of the bone flap, to avoid crushing the wires after bone flat fixation to the skull. Extension wires are then tunneled under the skin, passing behind the ear and then in the neck and are then connected to the stimulator (Implanted Pulse Generator, IPG) placed subcutaneously in the pectoral region subclaveraly. A drain 
is occasionally inserted in the epidural space or in the subcutaneous space (Pirotte et al 2009). The skin is closed with separated 2-0 Dermalon sutures. Staples are used for skin closure, but attention must be lent not to damage the wires. It must be noted that, while for several applications (e.g. Parkinson's disease) a one-shot lead+IPG implantation is the norm, several authors only implant the IPG after a test period (few days to 2-4 weeks), e.g. in chronic pain conditions. In this case, the lead is connected subcutaneously in the retromastoid region (and not inferiorly in the soft tissues of the neck, as the connector may break or erode through the skin with time) to a temporary cable that exits through the skin behind the ear (by way of a small incision) and is then plugged into an external stimulator. Whatever the results of the test period, the temporary cable exiting through the skin is cut in order to allow the skin hole to heal properly (Pirotte et al 2009).

A postoperative CT scan is mandatory to check the exact lead position by fusing CT images with preoperative MRI images and evince postoperative hemorrhage.

\subsection{Neuronavigated rTMS-guided Technique}

rTMS can guide lead positioning by evincing the cortical area that affords the best clinical outcome. When rTMS induces e.g. marked pain relief, neuronavigated rTMS can be used to check the exact site of stimulation and MEPs can also be used to check that this site corresponds to M1 (Fig.5.5) and whether or not the target corresponds to the somatotopic distribution. The Nexstim neuronavigated rTMS system allows very precise localization (Ruohonen and Karhu 2010). The central region can be identified by using the "peeling" function. This function provides brain images corresponding to layers parallel to the cortical surface (Fig.5.7A-B), thereby minimizing parallax errors, while facilitating identification of the various sulci and fissures of the region (Fig.5.7B-D). Neuronavigated rTMS therefore allows precise localization of the effective target (the upper limb M1 area is shown in Fig.5.7A). This same target is easy to identify (Fig.5.7D), although the "peeling” function is not yet available on intraoperative neuronavigation systems. Intraoperative electrophysiological testing is no longer necessary, as MEPs have already been recorded during neuronavigated rTMS sessions (Fig.5.5). The procedure is therefore easier to perform and much more reliable, as the target has been precisely determined prior to the operation. 

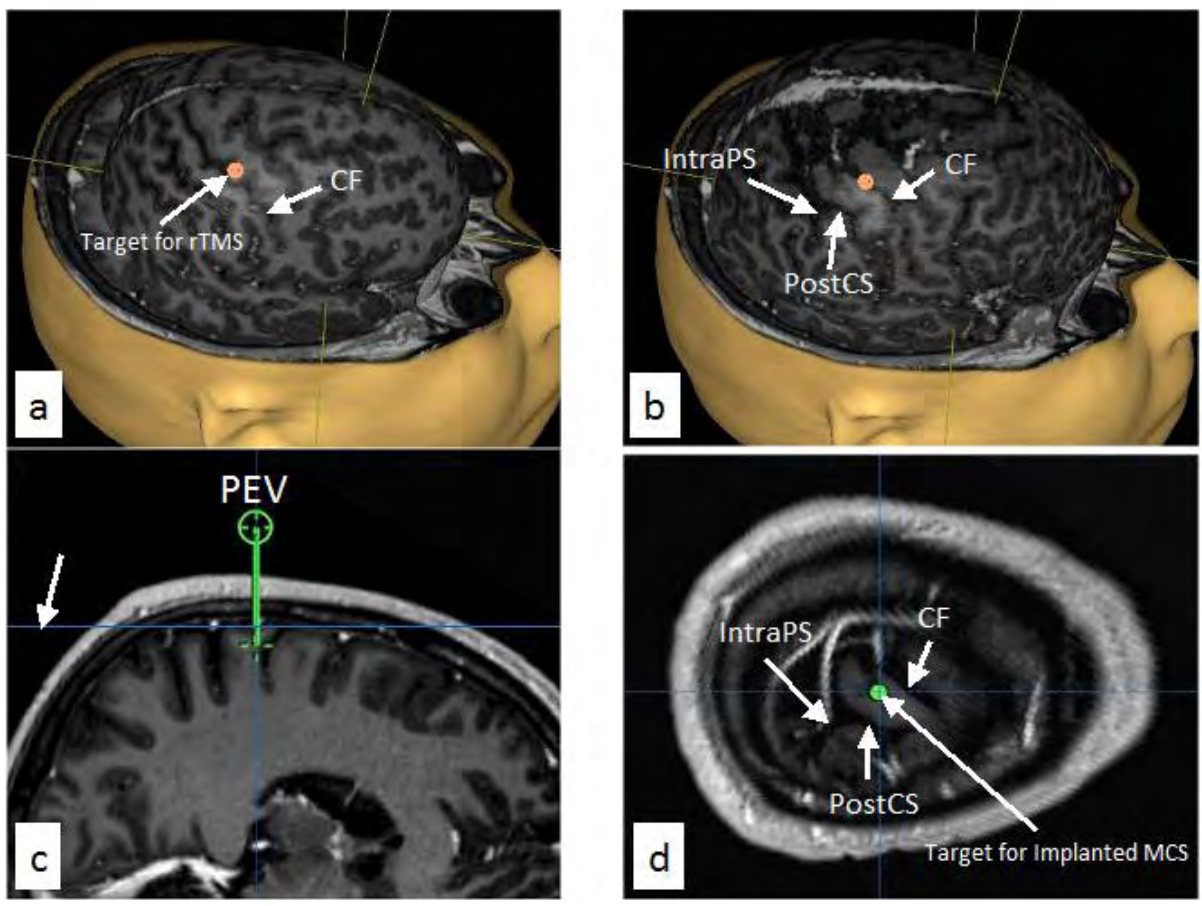

Fig. 5.7: Value of neuronavigated rTMS. Figures $a$ and $b$ show the position of the stimulation target shown to be effective on rTMS. It is situated just anteriorly to the central sulcus (CS Fig. a). Figure b: the same target is represented on the cortical surface. The postcentral sulcus (PostCS) and the intraparietal sulcus (Intra PS) can be easily identified. Figures $\mathrm{c}$ and d show the position of the target on neuronavigation. On Figure c, the arrow indicates the level of the section shown on Figure d. This Figure illustrates practically the same structures as those visible on Figure b. The target is placed slightly posteriorly to the central sulcus, just anteriorly to the intraparietal sulcus (Intra PS). Parallax errors can be minimized by carefully comparing neuronavigated rTMS data and intraoperative neuronavigation data.

\subsection{Subdural Positioning}

Some authors have proposed implanting leads in the subdural space, especially to treat pain in the lower limbs. The main disadvantage is that this is a relatively invasive technique with, in particular, a risk of injury to veins of the central region during positioning of leads on the medial surface of the hemisphere. It is also practically impossible to anchor the lead in a precisely determined position, which is associated with a risk of loss of efficacy due to lead displacement. The main publications describing this technique have reported disappointing results (e.g. Saitoh and Hosomi 2009). There may be a place for subdural lead placement in case of cerebral atrophy. However, results of ICS for Parkinson Disease and stroke rehabilitation clearly show 
that the lower limbs can be affected by stimulating the cortex outside the expected projection from Penfield's homunculus (see Chapters 10 and 12).

Some authors have recommended stimulating M1 directly by placing the electrode dead on the surface of the anterior bank of the CS. CS veins have been used as landmarks during subdural approaches, but, being sometimes located deep in the CS, cannot be identified by examining only the cortical surface. More relevant, large bridging veins sometimes interfere with implantation of the lead and adhesion may occur due to subarachnoid hemorrhage; dissection of the CS also involves the risk of developing new neurological deficits due to brain damage or vein obstruction. For upper limb and/or face pain, the arachnoid membrane of the CS must be carefully dissected and the vessels within it must be freed with a microsurgical procedure to expose the hidden lateral walls of the precentral and postcentral gyri. Since the paddle is too stiff to be placed within the CS, it must be trimmed off (Fig. 5.8). This approach is feasible only in patients with severe motor weakness or lack of function. Test stimulation of M1 within the CS is more effective in most cases than is subdural stimulation on the cerebral surface, but long-term clinical results are not on a par with extradural ICS. At the end of surgery, the lead extension is fixed to the dura or the border of the burr hole with a silk suture to prevent dislocation. However, migration of the electrodes seems to be more of a problem with a subdural than an extradural approach. A meticulous, watertight dural closure is mandatory to minimize the risk of cerebrospinal fluid leakage (Saitoh and Hosomi 2009). Stimulation by intradural electrodes is also associated with a high risk of seizures (Bezard et al 1999). Again, lead placement in the subdural space could be indicated in patients with cerebral atrophy who are markedly improved by rTMS.

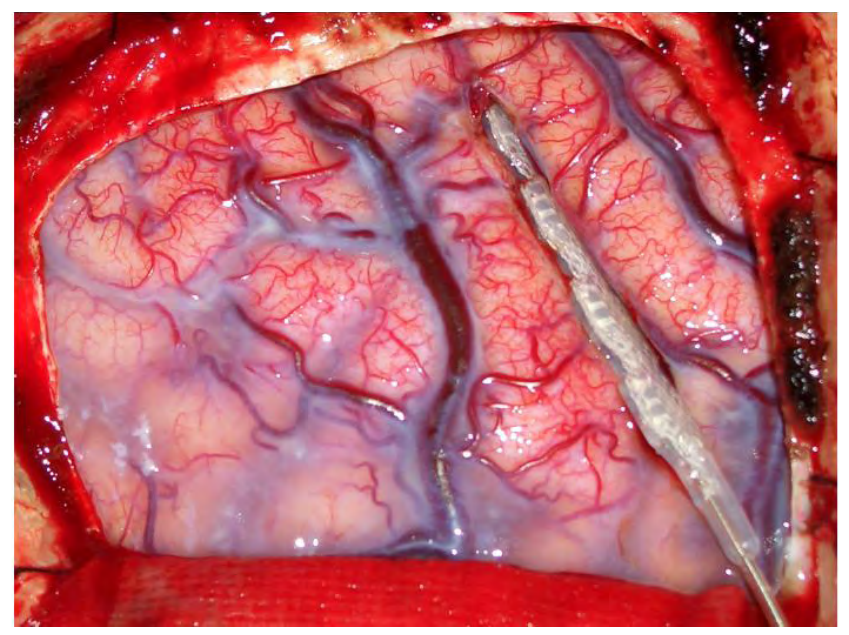

Fig. 5.8: Subdural lead placement. The quadripolar lead is inserted within the central sulcus after dissection of the arachnoid (From Saitoh and Hosomi 2009) 
A so-called interdural approach has been proposed: one or two dural strips, similar in size and shape to the actual strip electrode(s) are resected overlying the targeted area. The strip electrodes are then positioned in place of the resected dural strips, in a patchlike fashion and sutured to the edges of the dura. Finally the entire dural flap is reversed to its original position and sutured with many stitches back to the edges of the surrounding dura (Sakas et al 2011). It is not clear what the (supposed) advantages should be.

In sum, lead implantation procedure has become simpler with the use of neuronavigated rTMS, as intraoperative electrophysiological testing is no longer required. It has also become more precise and more effective because the precise stimulation target can be predetermined by rTMS.

\section{References}

Bezard E, Boraud T, Nguyen JP, et al. Cortical stimulation and epileptic seizure: a study of the potential risk in primates. Neurosurgery 1999; 45: 364-350.

Conte V, L’Acqua C, Rotelli S, et al. Bispectral index during asleep-awake craniotomies. J Neurosurg Anesthesiol. 2013; 25: 279-284.

Holsheimer J, Nguyen JP, Lefaucheur JP, et al. Cathodal, anodal or bifocal stimulation of the motor cortex in the management of chronic pain ? Acta Neurochir Suppl. 2007 ; 97: 57-66.

Nguyen JP, Lefaucheur JP, Decq P, et al. Chronic motor cortex stimulation in the treatment of central and neuropathic pain. Correlations between clinical, electrophysiological and anatomical data. Pain 1999; 82: 245-251.

Nguyen JP, Nizard J, Keravel Y, et al. Invasive brain stimulation for the treatment of neuropathic pain. Nat Rev Neurol. 2011; 7: 699-709.

Pirotte BJM, Voordecker P, Levivier M, et al. Principles of surgical implantation and complications avoidance. In: Canavero S (ed) Textbook of therapeutic cortical stimulation. New York: Nova Science, 2009, pp 33-44

Ruohonen J and Karhu J. Navigated transcranial magnetic stimulation. Neurophysiol Clin. 2010; 40: 7-17.

Saitoh Y, Hosomi K. From localization to surgical implantation. In: Canavero S (ed) Textbook of therapeutic cortical stimulation. New York: Nova Science, 2009, pp 17-32

Sakas DE, Flaskas TN, Panourias IG, et al. Bifocal cortical electrical stimulation for pain by interdural implantation of the electrode. J Neurosurg 2011; 114:180-5. 